\title{
Efectos de la Rumiación y la Preocupación en el Desarrollo de Sintomatología Depresiva y Ansiosa en Estudiantes Universitarios de la Ciudad de Concepción, Chile
}

\section{Depressive Rumination and Worry in the Development of Depressive and Anxious Symptoms in University Students}

\author{
Mauricio Pimentel \\ $\mathrm{y}$ \\ Félix Cova \\ Universidad de Concepción, Chile
}

(Rec: 9 de junio de 2009; Acep: 13 de octubre de 2010)

\begin{abstract}
Resumen
Tanto la rumiación como la preocupación constituyen procesos cognitivos repetitivos e improductivos. Diversas investigaciones han mostrado de manera sistemática sus consecuencias negativas en el bienestar emocional. La presente investigación evaluó la influencia de la rumiación depresiva (incluyendo sus subfactores: reflexión y rumiación negativa), y de la preocupación en el desarrollo de sintomatología depresiva y ansiosa en una muestra de 226 estudiantes universitarios. Se realizaron dos mediciones con un lapso de 1 mes entre la primera y la segunda. Se observaron asociaciones entre mayores niveles de rumiación depresiva y preocupación, por un lado, y mayores niveles de sintomatología depresiva y ansiosa por otro. Sin embargo, controlada estadísticamente la sintomatología depresiva o ansiosa inicial, sólo la rumiación depresiva mostró una relación significativa con la sintomatología evaluada en el seguimiento, exclusivamente con ansiedad. Se observó un rol mediador de la rumiación y preocupación en las diferencias de género en síntomas ansiosos y depresivos. Estos resultados muestran la necesidad de una consideración especial de los pensamientos repetitivos en los programas de prevención y tratamiento de la sintomatología emocional.

Palabras clave: Rumiación depresiva, preocupación rumiación negativa, reflexión.
\end{abstract}

\begin{abstract}
Both rumination and worry are repetitive and unproductive cognitive processes. Different studies have shown their negative impact on emotional wellbeing. This investigation evaluates the influence of depressive rumination (including its subfactors: reflection and negative rumination), and worry in the development of depressive symptoms in a sample of 226 college students. Two measurements were performed with a time lag of 1 month between the first and second. The results showed a relationship between high levels of depressive rumination and worry and high levels of depressive and anxious symptoms. However, having being statistically controlled the initial depressive or anxious symptomatology, only depressive rumination showed a significant relationship with the assessed symptomatology in the follow-up, exclusively with anxiety. It was observed a mediator role of rumination and concern in the differences of gender in anxious and depressive symptoms. These results stress the need for special consideration of these types of repetitive thoughts on preventive programs and treatment of depression.
\end{abstract}

Key words: Depressive rumination, worry, negative rumination, reflection.

Correspondencia: Mauricio Pimentel G., Facultad de Psicología, Universidad de Concepción, Concepción, Fono: (041) 2134700. E-mail: mopg7@ hotmail.com Investigación financiada por: Proyecto Fondecyt 11060059. 


\section{Introducción}

La investigación ha posibilitado apreciar la potencial contribución de la rumiación y de la preocupación sobre el desarrollo y mantención de síntomas y trastornos emocionales, especialmente sobre los síntomas y trastornos depresivos y ansiosos (Abela \& Hankin, 2009; Fresco, Frankel, Mennin, Turk \& Heimberg, 2002; Hankin, 2008). Sin embargo, pese a la estrecha vinculación conceptual entre rumiación y preocupación, la investigación al respecto ha tendido a ser realizada sin considerar esta interrelación. De hecho, los estudios sobre los efectos de la rumiación se han concentrado fundamentalmente en la depresión y los de preocupación en la ansiedad. Sólo recientemente se han abordado de modo más sistemático las posibles relaciones entre preocupación y rumiación (Nolen-Hoeksema, Wisco \& Lyubomirsky, 2008). Los resultados de tales estudios han ofrecido sustento para la conceptualización de la preocupación y de la rumiación como procesos distintos pero relacionados (Fresco et al., 2002; Nolen-Hoeksema et al., 2008; Watkins, Moulds \& Mackintosh, 2005). Algunos de estos estudios plantean la posibilidad que tanto la rumiación y la preocupación jueguen un papel más relevante en el desarrollo de los síntomas de ansiedad, contradiciendo el supuesto que la rumiación sería más específica de la depresión (Calmes \& Roberts, 2007; Segerstrom, Tsao, Alden \& Craske, 2000).

Nolen-Hoeksema ha sido una de las principales investigadoras respecto de la rumiación y la ha definido como "pensamientos repetitivos en el hecho de que uno está deprimido, en los síntomas depresivos y en las causas, significados y consecuencias de estos síntomas" (NolenHoeksema \& Morrow, 1991, p. 119). De allí que esta forma de rumiación reciba el nombre de rumiación depresiva. El instrumento que se ha empleado para evaluar esta forma de rumiación es el Ruminative Responses Scale RRS (Treynor, González \& Nolen-Hoeksema, 2003). En el marco de esta tradición de investigación, se ha encontrado sustento teórico para proponer dos subtipos de rumiación: la rumiación reflexiva (reflection) y la rumiación negativa (brooding). Se ha planteado que ambos subtipos de rumiación podrían tener distintas consecuencias (Kuyken, Watkins, Holden \& Cook, 2006; Roberts, Attkinsson \& Rossenblant, 1998; Treynor et al., 2003; Watkins, 2004). Algunos estudios han mostrado que sería la rumiación negativa la que tendría un rol más perjudicial en relación al desarrollo de sintomatología depresiva (Cova, Melipillán \& Rincón, 2009; Joormann, Dkane \& Gotlib, 2006; Nolen-Hoeksema et al., 2008).

Por otro lado, a partir de los estudios experimentales de Thomas Borkovec durante las dos últimas décadas, se ha generado una línea de investigación en torno al fenómeno de la preocupación (Papageorgiou, 2006; Prados, 2005). La preocupación ha sido definida como: "una cadena de pensamientos y de imágenes, cargadas de afectos negativos y relativamente incontrolables; representa una tentativa de entablar una solución mental de problemas en un asunto cuyo resultado es incierto pero que contiene la posibilidad de unos o más resultados negativos" (Borkovec, Robinson, Pruzinsky \& Depree, 1983, p. 10). Ejemplos de preocupación sería la atención recurrente en pensar en las potenciales implicaciones del despido de un trabajo u otras como: ¿qué pasaría si no consigo un ascenso?, ¿qué pasaría si fallo en mi examen? ¿qué pasaría si me enfermo? (Papageorgiou, 2006).

Aunque la preocupación ha sido considerada el sello característico del trastorno de ansiedad generalizado (TAG) (APA, 2003), se ha observado que es frecuente en todos los trastornos de ansiedad (Barlow, 1988) y también en la depresión (Molina, Borkovec, Peasley, \& Person, 1998; Starcevic, 1995). Más ampliamente, se ha observado relación entre la preocupación y el afecto negativo en un sentido amplio (Beck, Perkins, Holder, Robbins, Gray \& Allison, 2001). Asimismo, se ha observado que la preocupación constituiría un antecedente del afecto negativo y ansiedad más que una consecuencia de éstas (Gana, Martin \& Canouet, 2001; Segerstrom, et al., 2000).

Los estudios epidemiológicos de trastornos emocionales han encontrado diferencias de género en cuanto a la prevalencia de éstos. Sistemáticamente se han reportado tasas más altas de trastornos ansiosos y depresivos en mujeres que en hombres, fenómeno que es manifiesto a partir de la adolescencia (Cova, 2003; Hoffmann, Powlishta \& With, 2004; Wade, Cairney \& Pevalin, 2002; Zahn-Waxler, Crik, Shirtcliff \& Woods, 2006). Asimismo, múltiples estudios han encontrado diferencias de género en rumiación y preocupación; en su mayoría muestran que las mujeres puntúan más alto que los hombres en los diferentes instrumentos que se han utilizado para medir estos tipos de pensamiento. Algunos autores han explorado la posibilidad que las diferencias de género en depresión y en ansiedad sean mediadas, en algún grado, por la rumiación y la preocupación (Butler \& Nolen-Hoeksema, 1994; Hilt, McLaughlin \& Nolen-Hoeksema, 2010; Nolen-Hoeksema, Larson \& Grayson, 1999). Un estudio realizado con adolescentes chilenos encontró efectivamente un efecto mediador de la rumiación en la mayor presencia tanto de sintomatología depresiva como ansiosa en mujeres (Cova, Rincón \& Melipillán, 2007).

Considerando estos antecedentes, la presente investigación se propuso evaluar la relación tanto de la rumiación depresiva (y sus componentes de reflexión y rumiación depresiva) como de la preocupación con el desarrollo de sintomatología depresiva y ansiosa. Otro objetivo fue analizar el rol de la rumiación y la preocupación en las diferencias de género en depresión y ansiedad. 


\section{Método}

\section{Diseño}

Con el fin de poder estimar el rol de la rumiación y preocupación no sólo como correlatos sino como posibles variables explicativas del desarrollo de sintomatología emocional, se optó por evaluar a los participantes en forma prospectiva. Se realizaron dos evaluaciones. La segunda evaluación fue realizada un mes después de la primera.

\section{Participantes}

La muestra fue obtenida mediante un muestreo no probabilístico por accesibilidad y estuvo compuesta por 226 universitarios (96 hombres y 130 mujeres), de la Ciudad de Concepción, Sur de Chile, y cuyas edades fluctuaban entre los 18 y 28 años, con una media de 20,55 (DS 1,8). Se seleccionaron 5 cursos de diferentes carreras de 3 universidades diferentes, buscando que la muestra total tuviera distribución equitativa por sexo.

\section{Instrumentos}

\section{Escala de Respuesta Rumiativa (RRS):}

La escala utilizada en esta investigación corresponde a la versión abreviada de la Ruminative Response Scale (Treynor et al., 2003). En la RRS cada participante debe autoinformar la frecuencia con la que realiza las conductas indicadas por el instrumento en los momentos en que se encuentra triste o "bajoneado". Esta escala fue adaptada al español por Cova et al., (2007) y ampliada en cuatro ítem (Cova et al., 2009). Análisis factoriales y confirmatorios, tanto de la escala original como de la versión abreviada, indican que esta escala está compuesta por dos factores, de 5 ítem cada uno: Reflexión y Rumiación Negativa. Sus propiedades psicométricas se han mostrado adecuadas (Cova et al., 2007, 2009).

En el presente estudio se obtuvo un alfa total de $0.81 \mathrm{y}$ un 0.83 en la primera y segunda aplicación respectivamente. Para la subescala rumiación negativa el alfa fue de 0,81 en ambas aplicaciones, y de 0,69 y 0,70 para la subescala de reflexión.

Inventario de Preocupación de Pensilvania (PSWQ): El PSWQ (Meyer, Miller, Metzger \& Borkovec, 1990) es una medida de autoinforme; tiene 16 ítem que intentan recoger la frecuencia e intensidad de la preocupación en general así como la dificultad del sujeto para controlarla, sin hacer referencia alguna a contenidos concretos de preocupación.

La fiabilidad y validez del PSWQ, así como su capacidad predictiva sobre la presencia del Trastorno de Ansiedad Generalizada y su utilidad como medida del cambio terapéutico en este trastorno y como instrumento de screening o cribaje para su detección, han recibido un amplio y sólido apoyo en la población general. Su consistencia interna se sitúa en los diferentes estudios entre 0,88 y 0,95 (Startup \& Erickson, 2006). Brown, Anthony \& Barlow (1992), informan de un coeficiente de fiabilidad test-retest que oscila entre 0.74 y 0.92 y una consistencia interna de 0,95 .

En el presente estudio se obtuvo un alfa de 0,94 y un 0,95 en la primera y segunda aplicación respectivamente.

Inventario de Depresión de Beck-Segunda Versión (BDI-II): El Inventario de Depresión de Beck-Segunda Versión BDI-II (Beck, Steer \& Brown, 1996), es un instrumento de autoaplicación de 21 ítem. Ha sido diseñado para evaluar la gravedad de sintomatología depresiva en adultos y adolescentes con una edad mínima de 13 años. En cada uno de los ítems la persona tiene que elegir, entre un conjunto de cuatro alternativas ordenadas de menor a mayor gravedad, la frase que mejor describe su estado durante las últimas dos semanas incluyendo el día en que completa el instrumento. Beck et al. (1996) observaron una alta consistencia interna del BDI-II tanto en muestras clínicas como no clínicas, con un coeficiente alfa de alrededor de 0,92 . En la adaptación española realizada por el equipo de Sanz, el Inventario para la Depresión de Beck-II obtuvo niveles elevados de consistencia interna tanto en muestras de universitarios, de población general y de consultantes con trastornos psicopatológicos (Sanz, García, Espinosa, Fortún \& Vásquez, 2005; Sanz, Navarro \& Vázquez, 2003; Sanz, Perdigón \& Vásquez, 2003). Los análisis factoriales en estas investigaciones concordaron con los estudios de Beck en reconocer en el BDI-II una dimensión general de depresión compuesta por dos factores altamente relacionados, uno cognitivo-afectivo y otro somático-motivacional. Las propiedades psicométricas del BDI-II han sido estudiadas en adolescentes en Chile (Melipillán, Cova \& Rincón, 2008). La traducción del equipo de Beck et al., (1996), y la realizada en España por Sanz et al. (2003), fueron contrastadas entre sí y respecto del instrumento original en inglés. El inventario mostró un alto grado de consistencia interna (alfa $=0,91)$ y apropiada correlación test-retest $(\mathrm{t}=0,66)$, así como capacidad para identificar adolescentes consultantes en servicios de salud mental.

En el presente estudio se obtuvo un alfa de 0,87 y un 0,91 en la primera y segunda aplicación respectivamente.

Subescala para medir trastornos por ansiedad: Esta subescala pertenece a la Escala para medir trastornos depresivos, por ansiedad y somatomorfos (Alvarado \& Vera, 1990). Esta subescala evalúa la presencia de síntomas correspondientes al trastorno por ansiedad, tomando como referencia los criterios diagnósticos descritos en el DSM-III-R para dicho cuadro. Para el presente estudio se han utilizado 10 elementos de la escala que miden síntomas ansiosos. Los participantes clasifican los ítem utilizando una escala Likert de tres puntos: "Nunca" $=0$ puntos, "Pocas veces" $=1$ punto, "Frecuentemente" $=2$ puntos. La puntuación varía de 0 a 20 puntos. La mayor presencia de 
ansiedad es la suma de la puntuación bruta de los 10 ítem. En el presente estudio se obtuvo un alfa de 0,79 y un 0,84 en la primera y segunda aplicación respectivamente.

\section{Análisis de datos}

Los análisis se realizaron con el paquete estadístico SPSS 15.0. En primer lugar se realizó un análisis correlacional de las variables consideradas en el estudio, para el cual fue utilizado el Coeficiente de Correlación productomomento de Pearson (r).

En segundo lugar se efectuaron análisis de regresión lineal múltiple jerárquica. Mediante éstos se buscó estimar el efecto conjunto de las distintas variables consideradas en este estudio, así como determinar el efecto particular que cada variable ejerce sobre otra, al controlar la influencia de las restantes. Previo al análisis se aseguró el cumplimento de los supuestos estadísticos requeridos para estos procedimientos. Dada la no normalidad observada de la variable depresión, se trabajó con una variante normalizada de ésta.

\section{Resultados}

La tabla 1 expone algunas medidas descriptivas calculadas a partir de la muestra para ambas aplicaciones. Se puede observar la similitud que mantuvieron las diferentes escalas, tanto en la evaluación inicial como en su seguimiento, sobre los valores en la media y desviación estándar.

Se aprecia una tendencia de la muestra hacia valores bajos y muy bajos en la escala de depresión y ansiedad en ambas aplicaciones, como lo demuestra la media de los participantes. Así también lo demuestra la desviación promedio que presentan los sujetos de la muestra.

Con respecto a las variables independientes, éstas muestran una tendencia hacia valores medios en sus respectivas escalas, como se puede apreciar observando la media de los participantes. No obstante, la muestra mantiene una tendencia hacia valores bajos en la subescala de rumiación negativa.

Tabla 1.

Estadísticos por Escala

\begin{tabular}{lcc}
\hline Escalas & Media & D.E \\
\hline BDI T $_{1}$ & 13.45 & 8.61 \\
BDI T $_{2}$ & 12.79 & 9.87 \\
${\text { Ansiedad } \mathrm{T}_{1}}_{\text {Ansiedad T }}$ & 7.47 & 3.91 \\
RRS T $_{1}$ & 6.70 & 4.32 \\
RRS T & 31.72 & 7.15 \\
Rum. Negativa $\mathrm{T}_{1}$ & 29.85 & 7.02 \\
Rum.Negativa $\mathrm{T}_{2}$ & 14.98 & 4.60 \\
Reflexión $\mathrm{T}_{1}$ & 14.14 & 4.38 \\
Reflexión $\mathrm{T}_{2}$ & 16.73 & 3.88 \\
PSQW T & 15.72 & 3.77 \\
PSQW $\mathrm{T}_{2}$ & 31.23 & 14.17 \\
\hline D. Desvicion Estandar & 28.47 & 14.39 \\
\hline
\end{tabular}

D.E: Desviación Estándar

La rumiación depresiva mostró una alta asociación con sintomatología depresiva al momento de ser medida transversalmente $(0,55 \mathrm{p}<0,001)$ y una moderada asociación con el seguimiento al mes $(0,43 \mathrm{p}<0,001)$. De manera similar, presentó asociaciones moderadas con sintomatología ansiosa, tanto en forma transversal $(0,49 p<0,001)$, como prospectiva $(0,46 \mathrm{p}<0,001)$. Por otro lado, la preocupación mostró una alta asociación con sintomatología depresiva $(0,52 \mathrm{p}<0,001)$ y ansiosa $(0,0 \mathrm{p}<0,001)$ al momento de ser medida transversalmente y una moderada correlación con sintomatología depresiva $(0,35 \mathrm{p}<0,001)$ y ansiosa $(0,42 \mathrm{p}<0,001)$ en el seguimiento a un mes.

Se realizaron una regresión con depresión y otra con ansiedad evaluadas al mes de seguimiento como variables

dependientes, controlando en el paso 1 la sintomatología inicial (Tablas 1 y 2). En el paso 2 se ingresaron la rumiación depresiva y la ansiedad. Sólo se observó un incremento significativo de la varianza explicada al introducir las variables rumiación depresiva y preocupación respecto de la ansiedad $(\Delta \mathrm{R} 2=0,02)$, siendo la rumiación depresiva la única variable con un efecto significativo $(\beta=0,17 ; \mathrm{sr} 2=0,02)$. La preocupación no mostró ningún efecto específico sobre la sintomatología depresiva y ansiosa en su seguimiento a un mes al controlar estadísticamente la sintomatología inicial. Estos análisis fueron repetidos ingresando en las regresiones separadamente rumiación depresiva y preocupación para analizar si la covarianza entre estas variables estaba diminuyendo sus efectos, pero los resultados obtenidos no mostraron ningún cambio respecto de los señalados. 
Efectos de la Rumiación y la Preocupación en el Desarrollo de Sintomatología Depresiva y Ansiosa en

Estudiantes Universitarios de la Ciudad de Concepción, Chile.

Tabla 2.

Regresión lineal múltiple jerárquica para la variable depresión en su evaluación de seguimiento a un mes con género, rumiación depresiva y preocupación como predictores

\begin{tabular}{|c|c|c|c|c|c|}
\hline & & B & $\mathrm{EE}$ & $\beta$ & $\mathrm{sr}^{2}$ \\
\hline \multirow[t]{4}{*}{ Paso 1} & Constante & $0,06 * * *$ & 0,19 & & \\
\hline & DepresiónT $_{1}$ & $0,94 * * *$ & 0,05 & 0,79 & 0,58 \\
\hline & Género & $-0,08$ & 0,13 & $-0,03$ & 0,00 \\
\hline & $R=0,78 * * * ; R^{2}$ & & & & \\
\hline \multirow[t]{6}{*}{ Paso 2} & Constante & $-0,01$ & 0,29 & & \\
\hline & Depresión $T_{1}$ & $0,96 * * *$ & 0,07 & 0,81 & 0,41 \\
\hline & Género & $-0,05$ & 0,13 & $-0,02$ & 0,00 \\
\hline & Rumiación & 0,01 & 0,01 & 0,04 & 0,00 \\
\hline & Preocupación & $-0,01$ & 0,01 & $-0,08$ & 0,00 \\
\hline & $R=0,79 ; R^{2}=$ & & & & \\
\hline
\end{tabular}

$*: \mathrm{p}<0,05 ; * *: \mathrm{p}<0,01 ; * * *: \mathrm{p}<0,001$

Variable dependiente: Depresión $\mathrm{T}_{2}$

$B$ : Coeficiente de regresión no estandarizado, $E E$ : Error estándar, $\beta$ : Coeficiente de regresión estandarizado, $s r^{2}=$ Correlación semiparcial al cuadrado.

Tabla 3.

Regresión lineal múltiple jerárquica para la variable ansiedad en su evaluación de seguimiento a un mes con género, rumiación depresiva y preocupación como predictores

\begin{tabular}{|c|c|c|c|c|c|}
\hline & & $\mathrm{B}$ & $\mathrm{EE}$ & $\beta$ & $\mathrm{sr}^{2}$ \\
\hline \multirow[t]{4}{*}{ Paso 1} & Constante & $0,87 * * *$ & 0,45 & & \\
\hline & AnsiedadT $_{1}$ & $0,82 * * *$ & 0,06 & 0,74 & 0,48 \\
\hline & Género & $-0,56$ & 0,45 & $-0,07$ & 0,00 \\
\hline & $R=0,72 * * * ; R^{2}=0,52\left(R_{\text {ajustado }}^{2}=0,52\right)$ & & & & \\
\hline \multirow[t]{6}{*}{ Paso 2} & Constante & $-1,32 * *$ & 0,93 & & \\
\hline & Ansiedad $\mathrm{T}_{1}$ & $0,77 * * *$ & 0,07 & 0,71 & 0,28 \\
\hline & Género & $-0,50$ & 0,44 & $-0,06$ & 0,00 \\
\hline & Rumiación & $0,10 * * *$ & 0,04 & 0,17 & 0,02 \\
\hline & Preocupación & $-0,02$ & 0,02 & $-0,08$ & 0,00 \\
\hline & $R=0,74 ; R^{2}=0,54\left(R_{\text {ajustado }}^{2}=0,53\right)$ & & & & \\
\hline
\end{tabular}

$*: \mathrm{p}<0,05 ; * *: \mathrm{p}<0,01 ; * * *: \mathrm{p}<0,001$

Variable dependiente: Ansiedad $\mathrm{T}_{2}$

$B$ : Coeficiente de regresión no estandarizado, $E E$ : Error estándar, $\beta$ : Coeficiente de regresión estandarizado, $s r^{2}=$ Correlación semiparcial al cuadrado. 
Los dos subfactores de la rumiación depresiva (rumiación negativa y reflexión) correlacionaron con la sintomatología depresiva y ansiosa. La rumiación negativa presentó una alta correlación con sintomatología depresiva inicial $(0,59 \mathrm{p}<0,001)$ y una moderada correlación con sintomatología depresiva en su seguimiento a un mes $(0,48$ $\mathrm{p}<0,001)$. Igualmente, mostró correlaciones moderadas con sintomatología ansiosa inicial $(0,52 p<0,000)$ y en su seguimiento a un mes $0,49(\mathrm{p}<0,001)$. Por otro lado, la reflexión exhibió una correlación moderada con sintomatología depresiva inicial $(0,32 p<0,001)$ y una correlación muy débil con sintomatología depresiva en su seguimiento a un mes $(0.23 p<0,001)$. En cuanto a su asociación con la sintomatología ansiosa, ésta fue débil, tanto en la medición transversal $0,29(\mathrm{p}<0,001)$, como prospectiva 0,27 ( $\mathrm{p}<$ $0,001)$.

Se realizaron análisis de regresión considerando como predictores los dos subfactores de rumiación depresiva (rumiación negativa y reflexión), con depresión y ansiedad evaluadas al mes de seguimiento como variables dependientes. Se controló nuevamente en el paso 1 la sintomatología inicial. Este análisis mostró incremento significativo de la varianza explicada al introducir la rumiación negativa y reflexión sólo respecto de la ansiedad (Tabla 3), observándose un efecto significativo sólo de la rumiación negativa sobre la sintomatología ansiosa $(\beta=0,15 ; \mathrm{sr} 2=0,01)$. La rumiación negativa sólo mostró en consecuencia valor predictor respecto de la sintomatología ansiosa; la reflexión no mostró relación con el desarrollo de sintomatología depresiva ni tampoco ansiosa.
Para determinar la posible existencia de un efecto mediador de las variables rumiación depresiva y preocupación en la relación entre género y sintomatología depresiva, y entre género y sintomatología ansiosa, se realizó el procedimiento sugerido por Baron \& Kenny (1986). Este procedimiento implica en este contexto cuatro supuestos: la variable independiente (género) debe relacionarse de manera significativa con la variable dependiente (sintomatología ansiosa y depresiva). En segundo lugar, la variable independiente debe relacionarse de manera significativa con la variable mediadora (rumiación depresiva - preocupación). En tercer lugar, la variable mediadora debe relacionarse de manera significativa con la variable dependiente mientras se controla el efecto de la variable independiente (rumiación depresiva y preocupación deben relacionarse con depresión y ansiedad mientras se controla el efecto del género). Finalmente, la existencia de un efecto mediador queda demostrada si la variable independiente sobre la variable dependiente disminuye de manera significativa, o incluso desaparece, cuando la variable mediadora es incluida en el modelo.

Los análisis correspondientes a la evaluación del efecto mediador de rumiación en la relación entre género y depresión medida prospectivamente se presentan en la Tabla 4. Como se observa en esta tabla, las tres primeras condiciones se cumplieron adecuadamente. En cuanto a la cuarta condición, se apreció una reducción en el coeficiente de regresión correspondiente a la variable género desde un valor inicial de 0,51 , a un valor de 0,22 cuando se controló el efecto de la variable mediadora rumiación y preocupación.

Tabla 4.

Regresión lineal múltiple jerárquica para la variable ansiedad en su evaluación de seguimiento a un mes con género, rumiación negativa y reflexión como predictores

\begin{tabular}{|c|c|c|c|c|c|}
\hline & & $\mathrm{B}$ & $\mathrm{EE}$ & $\beta$ & $\mathrm{sr}^{2}$ \\
\hline \multirow[t]{4}{*}{ Paso 1} & Constante & 0,87 & 0,45 & & \\
\hline & Ansiedad $\mathrm{T}_{1}$ & $0,82 * * *$ & 0,06 & 0,74 & 0,48 \\
\hline & Género & $-0,56$ & 0,45 & $-0,07$ & 0,00 \\
\hline & $R=0,72 * * * ; R^{2}=0,52\left(R_{\text {ajustado }}^{2}=0,52\right)$ & & & & \\
\hline \multirow[t]{6}{*}{ Paso 2} & Constante & $-0,99$ & 0,96 & & \\
\hline & Ansiedad $\mathrm{T}_{1}$ & $0,72 * * *$ & 0,06 & 0,66 & 0,28 \\
\hline & Género & $-0,56$ & 0,44 & $-0,06$ & 0,00 \\
\hline & Rumiación Negativa & $0,14^{*}$ & 0,06 & 0,15 & 0,01 \\
\hline & Reflexión & 0,03 & 0,06 & 0,03 & 0,00 \\
\hline & $R=0,74 ; R^{2}=0,54\left(R_{\text {ajustado }}^{2}=0,53\right)$ & & & & \\
\hline
\end{tabular}

$*: \mathrm{p}<0,05 ; * *: \mathrm{p}<0,01 ; * * *: \mathrm{p}<0,001$

Variable dependiente: Ansiedad $\mathrm{T}_{2}$

$B$ : de regresión no estandarizado, $E E$ : Error estándar, $\beta$ : Coeficiente de regresión estandarizado, $s r^{2}=$ Correlación semiparcial al cuadrado.Coeficiente 
Los resultados correspondientes al efecto mediador de las variables rumiación y preocupación en la relación entre el género y la sintomatología ansiosa medida prospectivamente se presentan en la Tabla 5. Una vez más se aprecia que las tres primeras condiciones propuestas por Baron $\&$ Kenney (1986) fueron cumplidas satisfactoriamente. En lo que respecta a la última condición, esto es, la disminución en el valor del coeficiente de la variable predictora, se observó una reducción en el coeficiente de regresión correspondiente a la variable género desde un valor inicial de 1.80 a un valor final de 0.81 cuando se controló el efecto de las variables rumiación y preocupación.

Tabla 5.

Resultados del análisis de regresión para la evaluación del efecto mediador de las variables rumiación depresiva y preocupación en la relación entre género y sintomatología depresiva $T 2 a(N=226)$

\begin{tabular}{lcccc}
\hline & B & EE & Beta & t \\
\hline Dependiente: Depresión & & & & $19,13^{* * * *}$ \\
Constante & 2,93 & 0,15 & & $2,52^{*}$ \\
Género & 0,51 & 0,20 & 0,17 &
\end{tabular}

Dependiente: Rumiación depresiva

$\begin{array}{lcccc}\text { Constante } & 30,09 & 0,74 & & 40,54^{* * * *} \\ \text { Género } & 2,44 & 0,98 & 0,17 & 2,49^{*} \\ \text { Dependiente: Preocupación } & & & & \\ \text { Constante } & 26,40 & 1,43 & & 18,43^{* * *} \\ \text { Género } & 7,64 & 1,90 & 0,27 & 4,03^{* * *}\end{array}$

Dependiente: Depresión

\begin{tabular}{lcccc} 
Constante & 0,42 & 0,42 & & 1,01 \\
Género & 0,22 & 0,19 & 0,08 & 1,16 \\
Rumiación depresiva & 0,02 & 0,01 & 0,15 & 1,93 \\
Preocupación & 0,07 & 0,02 & 0,34 & $4,60^{* * * *}$ \\
\hline
\end{tabular}

${ }^{a}$ Depresión evaluada al mes de seguimiento

$*: \mathrm{p}<0,05 ; * *: \mathrm{p}<0,01 ; * * *: \mathrm{p}<0,001$

\section{Discusión}

Se encontraron evidencias que apoyan la relación entre mayores niveles de rumiación depresiva y preocupación por un lado, y mayores niveles de sintomatología depresiva y ansiosa por otro. Los resultados mostraron correlaciones que van de moderadas a altas, tanto de forma transversal como prospectivamente. Estos resultados están en concordancia con numerosas investigaciones previas que dan cuenta de la asociación entre los pensamientos repetitivos en forma de rumiación y preocupación con la presencia y desarrollo de sintomatología ansiosa y depresiva (Nolen-Hoeksema et al., 2008; Watkins, 2008).
El diseño utilizado permitió analizar, si controlando la sintomatología prexistente, la relación entre rumiación depresiva, preocupación y depresión y ansiedad se mantenían. Todavía existe poca claridad respecto de si la rumiación depresiva y preocupación constituyen fundamentalmente correlatos de la ansiedad o depresión o se les puede atribuir un rol causal. Los resultados obtenidos al implementar este control (que hace más exigente la posibilidad de encontrar asociaciones) sólo mostraron una asociación entre mayores niveles de rumiación depresiva y sintomatología ansiosa. Varios estudios sugieren la ventaja de la preocupación por sobre la rumiación depresiva como 
predictor de la sintomatología ansiosa y depresiva (Muris, Roelofs, Meester \& Boomsma, 2004); sin embargo, en el presente estudio la preocupación no resultó un factor predictor ni de la presencia de sintomatología depresiva ni de la ansiosa (controlada la sintomatología previa). La ausencia de valor predictivo de la preocupación respecto de la sintomatología ansiosa al mes de seguimiento, una vez controlada la sintomatología inicial, podría indicar que la preocupación es relevante particularmente para ciertas formas específicas de ansiedad. La multidimensionalidad de la ansiedad ha dificultado la existencia de instrumentos de evaluación de ésta con suficiente validez de contenido. En la presente investigación de utilizó un instrumento que mide sintomatología ansiosa en forma genérica y que cubre más manifestaciones somáticas de ansiedad que cognitivas.

En lo referente a la rumiación depresiva, su comportamiento en esta muestra fue coherente con estudios recientes que han utilizado población universitaria y plantean la posibilidad de que la rumiación y la preocupación jueguen un papel más directo en el desarrollo de los síntomas ansiosos que depresivos (Nolen-Hoeksema, 2000; Segerstrom et al., 2000). Los resultados arrojaron que específicamente el componente de rumiación negativa es el que predice de manera más consistente los síntomas ansiosos, no así el componente de reflexión. De acuerdo a estudios recientes, se piensa que la mayor reflexión puede ser una respuesta a la presencia de sintomatología emocional, y no ser necesariamente desadaptativa, a diferencia de la rumiación negativa que sí ha mostrado, en estudios prospectivos, una relación con el desarrollo posterior de depresión (Joorman et al., 2006; Treynor et al., 2003). Ni la rumiación depresiva ni la reflexión mostraron relación con el desarrollo de sintomatología depresiva, lo que es consistente con el hallazgo ya comentado respecto que el efecto negativo de la rumiación sobre la sintomatología emocional sería más directamente sobre la sintomatología ansiosa. La interdependencia de la sintomatología ansiosa y depresiva ha sido destacada largamente por la investigación, aun cuando los modelos explicativos de su interrelación todavía son controversiales. La propia delimitación de sintomatología depresiva y ansiosa no siempre es clara ni a nivel conceptual ni metodológico, lo que ha dificultado la investigación al respecto (Watson, 2009).

Los resultados derivados de esta investigación muestran la existencia de un efecto mediador de las variables rumiación y preocupación en la relación entre género y sintomatología depresiva, y entre género y sintomatología ansiosa. La mayor sintomatología depresiva y ansiosa observada en mujeres es parcialmente atribuible al efecto de estas variables. Ello indica que la existencia de mayor sintomatología emocional en mujeres puede ser explicada, entre otros factores, por el mayor empleo de estilos de pensamiento repetitivos negativos, como ha sido sugerido en diversas investigaciones (Hilt et al., 2010; Nolen-Hoeksema et al., 1999). Es de la mayor importancia identificar qué factores explican el mayor empleo de estos patrones repetitivos de pensamiento en el sexo femenino.

Los resultados obtenidos son de interés como indicios para una mayor comprensión del rol de los patrones de pensamiento repetitivos en el desarrollo de sintomatología emocional, y para el diseño de programa de tratamiento y prevención. La importancia de considerar las diferencias de género en estos patrones y su rol negativo en las mujeres es de particular interés. Se hace necesario que futuras estudios sean realizados en muestras más amplias y, especialmente, en tiempos de seguimiento más largos para darle mayor consistencia a los hallazgos presentados. 
Estudiantes Universitarios de la Ciudad de Concepción, Chile.

Tabla 6.

Resultados del análisis de regresión para la evaluación del efecto mediador de las variables rumiación depresiva y preocupación en la relación entre género y sintomatología ansiosa $T 2^{a}(N=226)$

\begin{tabular}{lcccc}
\hline & B & EE & Beta & t \\
\hline Dependiente: Ansiedad T & & & & \\
Constante & 5,57 & 0,44 & & $12,54^{* * *}$ \\
Género & 1,80 & 0,59 & 0,21 & $3,07^{* *}$
\end{tabular}

Dependiente: Rumiación depresiva

\begin{tabular}{|c|c|c|c|c|}
\hline Constante & 30,09 & 0,74 & & $40,54^{* * * *}$ \\
\hline Género & 2,44 & 0,98 & 0,17 & 2,49 \\
\hline \multicolumn{5}{|c|}{ Dependiente:Preocupación } \\
\hline Constante & 26,40 & 1,43 & & $18,43^{* * *}$ \\
\hline Género & 7,64 & 1,90 & 0,27 & $4,03^{* * *}$ \\
\hline \multicolumn{5}{|c|}{ Dependiente: Ansiedad $T^{2}$} \\
\hline Constante & $-2,12$ & 0,17 & & $-1,81$ \\
\hline Género & 0,81 & 0,54 & 0,09 & 1,51 \\
\hline Rumiación depresiva & 0,20 & 0,04 & 0,31 & $4,59 * * *$ \\
\hline Preocupación & 0,07 & 0,02 & 0,22 & $3,00 * *$ \\
\hline
\end{tabular}

a Ansiedad evaluada al mes de seguimiento

$*: \mathrm{p}<0,05 ; * *: \mathrm{p}<0,01 ; * * *: \mathrm{p}<0,001$

\section{Referencias}

Abela, J. R. Z., \& Hankins, B. (2009). Cognitive vulnerability to depression in adolescent: A developmental psychopathology perspective. En: S. Hoeksema (Ed.), Handbook of Depression in Adolescents (335-376). New York: Routledge.

Alvarado, R., \& Vera, A. (1990). Validación de escalas para medir trastornos depresivos, por ansiedad y somatomorfes. Cuadernos de trabajo $\mathrm{N}^{\circ}$ 2, Escuela de Psicología, Universidad Diego Portales, Santiago, Chile.

American Psychiatric Association. (2003). DSM-IV-TR: Brevario: Criterios Diagnósticos. Barcelona: Mason.

Baron, R., \& Kenny, D. (1986). The moderator-mediator variable distinction in social psychological research: Conceptual, strategic, and statistical considerations. Journal of Personality and Social Psychology, 51, 1173-1182.

Barlow, D. (1988). Anxiety and its disorders: The nature and treatment of anxiety and panic. New York: Guilford.

Beck, R., Perkins, T.S., Holder, R., Robbins, M., Gray, M., \& Allison, S.H. (2001). The cognitive and emotional phenomenology of depression and anxiety: Are worry and hopelessness the cognitive correlates of NA and PA? Cognitive Therapy and Research, 25, 819-827.
Beck, A.T., Steer, R.A., \& Brown, G.K. (1996). Manual for de Beck Depression Inventory, Second Edition. San Antonio, TX: The Psychological Corporation.

Borkovec, R., Robinson, E., Pruzinsky, T., \& Depree, J. (1983). Preliminary exploration of worry: Some characteristics and processes. Behaviour Research and Therapy, 21, 9-16.

Brown, T., Anthony, M., \& Barlow, D. (1992). Psychometry properties of the Penn State Worry Questionnaire in a clinical disorders sample. Behaviour Research and Therapy, 30, 33-37.

Butler, L. D., \& Nolen-Hoeksema, S. (1994). Gender differences in response to depressed mood in a college sample. Sex Roles, 30, 331-346.

Calmes, C., \& Roberts, J. (2007). Repetitive thought and emocional distress: Rumination and worry as prospective predictors of depressive and anxious symptomatology. Cognitive Therapy and Research, 30, 343-356.

Cova, F. (2003). Influencia familiar y diferencias de género en conductas externalizadas e internalizadas en preadolescentes. Tesis doctoral para optar al título de doctor en Psicología, Universidad del País Vasco, España.

Cova, F., Melipillán, R., \& Rincón, P. (2007). Rumiación y presencia de sintomatología ansiosa y depresiva en adolescentes. Revista Mexicana de Psicología, 24, 175-183. 
Cova, F. Melipillán, R., \& Rincón, P. (2009). Reflexión, rumiación negativa y desarrollo de sintomatología depresiva en adolescentes de sexo femenino. Terapia Psicológica, 27, 155-160.

Fresco, D., Frankel, A., Mennin, D., Turk, C., \& Heimberg, R. (2002). Distinct and overlapping features of rumination and worry: The relationship of cognitive production to negative affective states. Cognitive Therapy and Research, 26, 179-188.

Gana, K., Martin, B., \& Canouet, M.D. (2001). Worry and anxiety: Is there a causal relationship? Psychopathology, 34, 221-229.

Hankin, B. (2008). Rumination and Depression in Adolescence: Investigating Symptom Specificity in a Multiwave Prospective Study. Journal of Clinical Child \& Adolescent Psychology. 37, $701-713$.

Hilt, L., McLaughlin, K., \& Nolen-Hoeksema, S. (2010). Examination of the response styles theory in a community sample of young adolescents. Journal of Abnormal Child Psychology, 38, 545-556.

Hoffmann, M., Powlishta, K., \& With, K. (2004). An examination of gender differences in adolescent adjustment: The effect of competence on gender. Sex Roles, 50, 795-811.

Joorman, J., Dkane, M., \& Gotlib, I. (2006). Adaptive and maladptive componenents of rumination? Diagnostic specificity and relation to depressive biases. Behavior Therapy, 37, 269-280.

Kuyken, W., Watkins, E., Holden, E., \& Cook, W. (2006). Rumination in adolescents at risk for depression. Journal of Affective Disorders, 96, 39-47.

Melipillán, R., Cova, F., Rincón, P., \& Valdivia, M. (2008). Propiedades Psicométricas del Inventario de Depresión de Beck-II en Adolescentes Chilenos. Terapia Psicológica, 26, 59-70.

Meyer, T., Miller, M., Metzger, R., \& Borkovec, T. (1990). Development and validation of the Penn State Worry Questionnaire. Behaviour Research and Therapy, 28, 487-495.

Molina, S., Borkovec, T., Peasley, C., \& Person, D. (1998). Content Analysis of Worrisome Streams of Consciousness in Anxious and Dysphoric Participants. Cognitive Therapy and Research, 22, 109-123.

Muris, P., Roelofs, J., Meester, C., \& Boomsma. (2004). Rumination and worry in nonclinical adolescents. Cognitive Therapy and Research, $28,539-554$.

Nolen-Hoeksema, S. (2000). The role of rumination in depressive disorders and mixed anxiety/depressive symptoms. Journal of Abnormal Psychology, 109, 504-511.

Nolen-Hoeksema, S., Larson, J., \& Grayson, C. (1999). Explaining the gender difference in depressive symptoms. Journal of Personality and Social Psychology, 77, 101-107.

Nolen-Hoeksema, S., \& Morrow, J. (1991). A prospective study of depression and posttraumatic stress symptoms after a natural disaster: The 1989 Loma Prieta earthquake. Journal of Personality and Social Psychology, 61, 115-121.

Nolen-Hoeksema, S., Wisco, B., \& Lyubomirsky, S. (2008). Rethinking rumination, Perspectives on Psychological Science, 3, 400-424.
Papageorgiou, C. (2006). Worry and rumination: Styles of persistent negative thinking in anxiety and depression. En G. Davey \& A. Wells (Eds.), Worry and its psychological disorders: Theory, assessment and treatment (21-40). England: Wiley.

Prados, M. (2005). El uso científico del término preocupación. Ansiedad y Estrés, 11, 181-191.

Roberts, R., Attkinsson, C., \& Rossenblant, A. (1998). Prevalence of psychopathology among children and adolescent. American Journal of Psychiatry, 155, 715-725.

Sanz, J., García, M.P., Espinosa, R., Fortún, M., \& Vázquez, C. (2005). Adaptación española del Inventario para la Depresión de Beck-II (BDI-II): 3. Propiedades psicométricas en pacientes con trastornos psicológicos. Clínica y Salud, 16, 121-142.

Sanz, J., Navarro, M.E., \& Vázquez, C. (2003). Adaptación española del Inventario para la Depresión de Beck-II (BDI-II): 1. Propiedades psicométricas en estudiantes universitarios. Análisis y Modificación De Conducta, 29, 239-288.

Sanz, J., Perdigón, A., \& Vázquez, C. (2003). Adaptación española del Inventario para la Depresión de Beck-II (BDI-II): 2. Propiedades psicométricas en población general. Clínica y Salud, 14, 249-280.

Segerstrom, S.C., Tsao, J.C.I., Alden, L.E., \& Craske, M.G. (2000). Worry and rumination: Repetitive thought as a concomitant and predictor of negative mood. Cognitive Therapy and Research, 24, 671-688.

Startevic, V. (1995). Anxiety and academic performance: A meta-analysis of findings. Anxiety Research, 4, 27-41.

Startup, H., \& Erickson, T. (2006). The Penn State Worry Questionnairse. En G. Davey \& A. Wells (Eds.), Worry and its psychological disorders: Theory, assessment and treatment (101-120). England: Wiley.

Treynor, W., González, R., \& Nolen-Hoeksema, S. (2003). Rumiation reconsidered: A psychometric analysis. Cognitive Therapy and Research, 27, 247-259.

Wade, T., Cairney, J., \& Pevalin, D. (2002). Emergence of gender differences in depression during adolescence: National panel results from three countries. Journal of American Academy of Child and Adolescent Psychiatry, 41, 190-198.

Watkins, E. (2004). Adaptative and maladaptive ruminative self-focus during emotional processing. Behaviour Research and Therapy, 42, 1037-1052.

Watkins, E. (2008). Constructive and unconstructive repetitive thought. Psychological Bulletin, 134, 163-206.

Watkins, E., Moulds, M., \& Mackintosh, B. (2005). Comparisons between rumination and worry in a non-clinical population. Behaviour Research and Therapy, 43, 1577-1585.

Watson, D. (2009). Differentiating the mood and anxiety disorders: A quadripartite model. Annual Review of Clinical Psychology, 5, 221-247.

Zahn-Waxler, C., Crik, N., Shirtcliff, E., \& Woods, E. (2006). The origin and development of psychopathology in females and males. En D. Cichetti, \& D. Cohen (Eds.), Developmental Psychopathology, Vol. I. ( $2^{\text {nd }}$ ed.) (pp.76-138), New Jersey: Wiley and Sons. 